\section{(6) OPEN ACCESS}

\title{
Lesinurad in combination with allopurinol: results of a phase 2 , randomised, double-blind study in patients with gout with an inadequate response to allopurinol
}

\author{
Fernando Perez-Ruiz, ${ }^{1}$ John S Sundy, ${ }_{1}^{2,3}$ Jeffrey N Miner, ${ }^{4}$ Matthew Cravets, ${ }^{4,5}$ \\ Chris Storgard, ${ }^{4}$ for the RDEA594-203 Study Group
}

\begin{abstract}
Handling editor Tore K Kvien
- Additional material is published online only. To view please visit the journal online (http://dx.doi.org/10.1136/ annrheumdis-2015-207919).

${ }^{1}$ Rheumatology Division, Hospital Universitario Cruces, Barakaldo, Spain

${ }^{2}$ Department of Medicine, Duke University Medical

Center, Durham, North Carolina, USA

${ }^{3}$ Gilead Sciences, Foster City, California, USA

${ }^{4}$ Ardea Biosciences, San Diego, California, USA

${ }^{5}$ Receptos, San Diego,

California, USA
\end{abstract}

\section{Correspondence to} Dr Chris Storgard, Ardea Biosciences, 9390 Town Center Drive, San Diego CA 92122,

USA; CStorgard@ardeabio.com

Received 13 May 2015 Revised 11 November 2015 Accepted 14 December 2015 Published Online First 7 January 2016

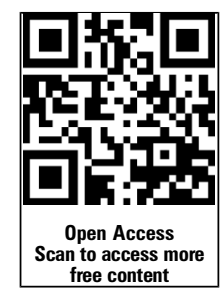

CrossMark

To cite: Perez-Ruiz F, Sundy JS, Miner JN, et al. Ann Rheum Dis

2016;75:1074-1080.

\section{ABSTRACT}

Objectives To assess the efficacy and tolerability of lesinurad, an oral selective uric acid reabsorption inhibitor, in combination with allopurinol versus allopurinol alone in patients with gout and an inadequate response to allopurinol.

Methods Patients $(\mathrm{N}=227)$ with an inadequate response to allopurinol, defined as serum urate (sUA) $\geq 6 \mathrm{mg} / \mathrm{dL}$ on $\geq 2$ occasions $\geq 2$ weeks apart despite $\geq 6$ weeks of allopurinol, were randomised 2:1 to 4 weeks of double-blind treatment with lesinurad $(200$ 400 or $600 \mathrm{mg} /$ day) or matching placebo in combination with their prestudy allopurinol dose (200-600 mg/day). Colchicine prophylaxis for gout flares was required. The primary end point was percent reduction from baseline sUA levels at 4 weeks. A pharmacokinetic substudy was also conducted. Safety was assessed throughout.

Results Patients ( $n=208$ ) received $\geq 1$ dose of blinded medication. Lesinurad 200, 400 and $600 \mathrm{mg}$ in combination with allopurinol produced significant mean percent reductions from baseline sUA of $16 \%, 22 \%$ and $30 \%$, respectively, versus a mean $3 \%$ increase with placebo ( $p<0.0001$, all doses vs placebo). Similar results were observed in patients with mild or moderate renal insufficiency (estimated creatinine clearance 30 to $<90 \mathrm{~mL}$ / min). The incidence of $\geq 1$ treatment-emergent adverse event was 46\%, 48\% and 54\% with lesinurad 200, 400 and $600 \mathrm{mg}$, respectively, and $46 \%$ with placebo (most frequent, gout flares, arthralgia, headache and nasopharyngitis), with no deaths or serious adverse events. Conclusions Lesinurad achieves clinically relevant and statistically significant reductions in SUA in combination with allopurinol in patients who warrant additional therapy on allopurinol alone.

Trial registration number NCT01001338.

\section{INTRODUCTION}

Gout is a monosodium urate (MSU) crystal deposition disease, resulting, if untreated, in chronic inflammatory arthritis that affects $1 \%-4 \%$ of adults. ${ }^{12}$ Elevated serum urate (sUA) levels (hyperuricaemia) lead to the formation of MSU crystal deposits within joint structures and soft tissues, leading to episodes of acute inflammation in articular and periarticular structures and tophus formation. ${ }^{3}$ In most individuals with gout, hyperuricaemia results from inefficient renal uric acid clearance. ${ }^{4}$ Targeting both uric acid production and inefficient excretion ${ }^{5}{ }^{6}$ with rational combination therapy can reduce sUA levels to achieve therapeutic goals. $^{7}$

Guidelines recommend a target sUA level of $<6 \mathrm{mg} / \mathrm{dL}$ (American College of Rheumatology $(\mathrm{ACR})$ ) or $\leq 6 \mathrm{mg} / \mathrm{dL}$ (European League Against Rheumatism (EULAR)) in patients with gout. ${ }^{5} 6$ A lower target (eg, <5) may benefit some patients, such as those with tophaceous gout. ${ }^{5} 6$ Available urate-lowering medications ${ }^{8}$ include the xanthine oxidoreductase (XO) inhibitors allopurinol and febuxostat, the urate transporter 1 (URAT1) inhibitors benzbromarone and probenecid, and the uricase enzyme pegloticase for severe gout. $^{9} 10$ Both the ACR and EULAR recommend titrated dosing of $\mathrm{XO}$ inhibitors as first-line urate-lowering therapy. ${ }^{5} 6$ However, a significant number of patients do not achieve the recommended sUA target. ${ }^{11-13}$ Thus, combination therapy adding uricosurics is suggested in patients failing $\mathrm{XO}$ inhibitors. $^{5}$

Lesinurad (RDEA594) is a selective uric acid reabsorption inhibitor (SURI) that inhibits the URAT1 transporter responsible for the majority of the reabsorption of filtered uric acid from the renal tubular lumen. ${ }^{14}{ }^{15}$ By inhibiting URAT1, lesinurad increases uric acid excretion and thereby lowers sUA. This trial was conducted to evaluate the efficacy and safety of lesinurad in combination with allopurinol in patients with gout and an insufficient urate-lowering response on allopurinol monotherapy.

\section{METHODS}

\section{Study design}

This was a randomised, double-blind, placebocontrolled trial (NCT01001338) conducted at sites in Canada $(n=6)$, Georgia $(n=2)$, Poland $(n=6)$, Spain $(n=2)$, Ukraine $(n=6)$, the UK $(n=2)$ and the USA ( $n=30)$ from 5 November 2009 to 27 January 2011. It contained a 3 -week screening period (including a 2-week run-in), a 4-week double-blind treatment period and a 2-week follow-up period. A substudy compared the multiple-dose pharmacokinetics (PK) of allopurinol and the active metabolite oxypurinol, with and without lesinurad coadministration.

The study protocol was reviewed and approved by the institutional review board/independent 
ethics committee at each site, and the study was conducted in accordance with the Declaration of Helsinki and the International Conference on Harmonisation/Good Clinical Practice. All patients provided written informed consent before participation.

\section{Patients}

Patients were men and postmenopausal or surgically sterile women 18-80 years of age with gout according to preliminary criteria for the classification of the acute arthritis of primary gout ${ }^{16}$ inadequate urate-lowering response (sUA $\geq 6 \mathrm{mg} / \mathrm{dL}$ on $\geq 2$ occasions $\geq 2$ weeks apart) to allopurinol monotherapy 200 $600 \mathrm{mg}$ daily for $\geq 6$ weeks and sUA $\geq 6 \mathrm{mg} / \mathrm{dL}$ at screening. Main exclusion criteria were history of documented or suspected kidney stones; active liver disease or clinically significant hepatic dysfunction; history of cardiac abnormalities; long-term use of salicylates $>100 \mathrm{mg}$ daily, thiazide diuretics (except $\leq 25 \mathrm{mg}$ hydrochlorothiazide), losartan, azathioprine, mercaptopurine, theophylline, intravenous colchicine, ciclosporin, cyclophosphamide, pyrazinamide, sulfamethoxazole or trimethoprim; uncontrolled hypertension; serum creatinine $(\mathrm{Cr})$ $>1.5 \mathrm{mg} / \mathrm{dL}$ or creatinine clearance $(\mathrm{CrCl})<60 \mathrm{~mL} / \mathrm{min}$ by Cockcroft-Gault formula using actual body weight (during the study, this protocol was amended to use ideal body weight) and body mass index $>48 \mathrm{~kg} / \mathrm{m}^{2}$.

\section{Treatment}

All patients received allopurinol at their prestudy dose (200$600 \mathrm{mg} /$ day $)$. To reduce gout flares, colchicine $(0.5$ or $0.6 \mathrm{mg} /$ day depending on local label and regulations) was given 14 days before baseline through week 1 of follow-up. Patients were assigned a randomisation number by time and date of request and randomised 2:1 within sequentially enrolled dose cohorts to 4 weeks of double-blind treatment with lesinurad once daily or placebo, respectively (figure 1 ). Colchicine-intolerant patients were not randomised. Following analysis of the initial cohort that received lesinurad $200 \mathrm{mg}$ daily or placebo, a $400 \mathrm{mg}$ daily cohort (200 mg for 7 days followed by $400 \mathrm{mg}$ for 21 days) and $600 \mathrm{mg}$ daily cohort $(200 \mathrm{mg}$ for 7 days followed by $400 \mathrm{mg}$ for 7 days and $600 \mathrm{mg}$ for 14 days) were added, and additional patients were added to the $200 \mathrm{mg}$ dose cohort.

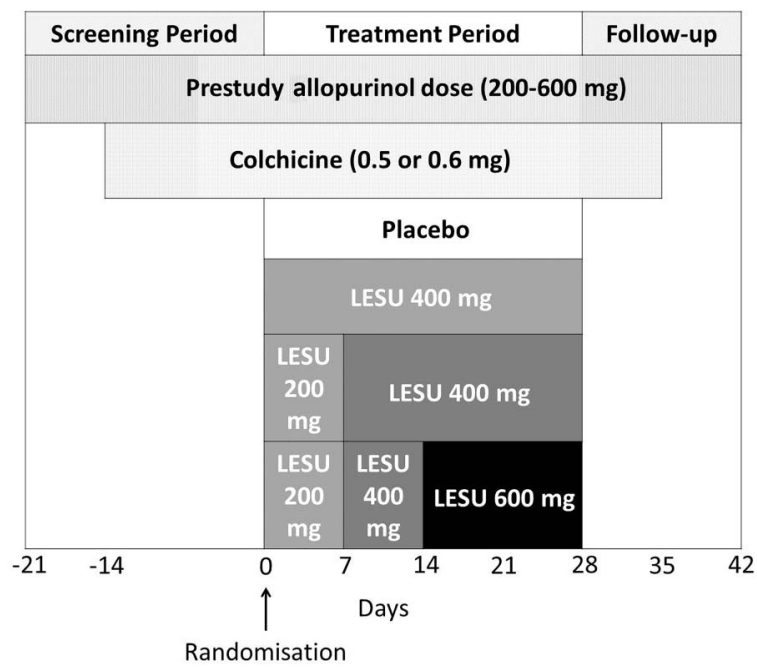

Figure 1 Study design. Patients received lesinurad $200 \mathrm{mg}$ daily or placebo for 28 days, $400 \mathrm{mg}$ daily ( $200 \mathrm{mg}$ for 7 days followed by $400 \mathrm{mg}$ for 21 days) or $600 \mathrm{mg}$ daily ( $200 \mathrm{mg}$ for 7 days followed by $400 \mathrm{mg}$ for 7 days and $600 \mathrm{mg}$ for 14 days). LESU, lesinurad.
Patients were randomised using a web-based central randomisation procedure, stratified by their prestudy allopurinol dose $(<300,300$ and $>300 \mathrm{mg})$. Patients, investigators, clinical staff and sponsor were blinded to treatment and sUA levels after the run-in period.

\section{Efficacy evaluations}

Patients underwent $24 \mathrm{~h}$ urine collections conducted at baseline and day 28 for assessment of sUA and renal handling of UA. The primary efficacy end point was percent reduction from baseline in sUA levels after 4 weeks in the intention-to-treat (ITT) cohort. Secondary efficacy end points included the proportion of patients with sUA $<6,<5$ and $<4 \mathrm{mg} / \mathrm{dL}$ at each visit; absolute (data not shown) and percent reduction in sUA from baseline at each visit and percent change from baseline in $24 \mathrm{~h}$ urine UA after 4 weeks.

\section{Safety and tolerability}

Safety was assessed using treatment-emergent adverse events (TEAEs), clinical laboratory tests, vital signs, 12-lead ECGs and physical examinations. Gout flares were collected as AEs and identified as requiring treatment if associated with concomitant medication.

\section{Pharmacokinetic analyses}

Details regarding PK analyses are provided in online supplementary material. A subset of patients consented to participate in a PK substudy, providing serial plasma and urine samples for analysis of allopurinol, oxypurinol, colchicine and lesinurad. They had an additional $24 \mathrm{~h}$ urine collection concluding on day 14 .

\section{Statistical analyses}

Data from the placebo groups within each cohort of lesinurad 200, 400 and $600 \mathrm{mg}$ were combined to provide more robust assessment of uric acid changes. The primary efficacy end point was analysed using analysis of covariance (ANCOVA) with effects for treatment group and baseline sUA. Each lesinurad group was compared with the pooled placebo group, and associated $\mathrm{p}$ values and $95 \%$ CIs for differences in least squares means are presented. No comparisons were made between lesinurad groups. Absolute and percent change from baseline in sUA at each visit and $24 \mathrm{~h}$ urine parameters (UA concentration, clearance and fractional excretion (FEur, UA clearance/ $\mathrm{CrCl} \times 100$ or FEur $=($ urine urate $\times$ serum $\mathrm{Cr}) /($ serum urate $\times$ urine $\mathrm{Cr}) \%)$ ) were analysed using methods for the primary analysis; within-cohort comparisons were performed using separate ANCOVAs. Proportions of patients with sUA $<6,<5$ and $<4 \mathrm{mg} / \mathrm{dL}$ were compared with Fisher's exact test. Missing data at day 27 were handled using a non-responder imputation analysis (patients with a missing day 27 sUA value were considered nonresponders) and a last observation carried forward (LOCF) analysis. Post hoc subgroup analyses were performed for patients with baseline estimated $\mathrm{CrCl}$ (Cockcroft-Gault formula using ideal body weight) $\geq 90 \mathrm{vs}<90 \mathrm{~mL} / \mathrm{min}$ (normal renal function vs renal insufficiency); with baseline sUA $<8,8$ to $<10, \geq 10$ and $\geq 8 \mathrm{mg} / \mathrm{dL}$ and with initial allopurinol dose $<300$ and $\geq 300 \mathrm{mg}$. Safety and PK end points were analysed using descriptive statistics.

Initially, a sample size of 36 patients per cohort was required to assess differences in proportions of patients with sUA $<6 \mathrm{mg} / \mathrm{dL}$. After external statistician review of results for the first cohort to determine sample size adequacy for the remaining cohorts, the primary end point changed to percent change in sUA per approved protocol amendment. Power analysis 
determined that 48 patients in the lesinurad group and 72 in the pooled placebo group were required for $80 \%$ power at $\alpha=0.05$ to detect a mean between-group difference of $12.6 \%$ for the primary end point. For within-cohort comparisons, 48 patients in the lesinurad group and 24 in the placebo group were needed for $80 \%$ power at $\alpha=0.05$ to detect a mean between-group difference of $17 \%$ for the primary end point.

Efficacy analyses were performed in the ITT population (all randomised patients who received $\geq 1$ dose of double-blind medication). To compare results with previous publications, LOCF was also used. Safety and tolerability were analysed in all patients who received double-blind medication based on actual treatment received.

\section{RESULTS}

\section{Patients}

Patients were recruited from 5 November 2009 to 3 November 2010; 227 were randomised (151 to lesinurad/allopurinol, 76 to allopurinol/placebo), and 208 received blinded study drug (safety and ITT populations). Of these, 136 received a combination of lesinurad/allopurinol (200 mg, $\mathrm{n}=46 ; 400 \mathrm{mg}, \mathrm{n}=42$; $600 \mathrm{mg}, \mathrm{n}=48$ ) and 72 received allopurinol alone (see online supplementary figure S1). Baseline demographics and clinical characteristics were similar across groups (table 1).

Using a Cockcroft-Gault formula with ideal body weight, $3 \%-7 \%$ of patients across groups had $\mathrm{CrCl} 30-59 \mathrm{~mL} / \mathrm{min}$ (table 1), whereas using actual body weight, as was done when screening patients for inclusion, one patient had $\mathrm{CrCl} \mathrm{30-}$ $59 \mathrm{~mL} / \mathrm{min}$ (chronic kidney disease stage 3). More patients had an initial allopurinol dose $\geq 300 \mathrm{mg}$ (lesinurad, $\mathrm{n}=114$; placebo, $\mathrm{n}=60$ ) than $<300 \mathrm{mg}$ (lesinurad, $\mathrm{n}=22$; placebo, $\mathrm{n}=12$ ).

\section{Primary efficacy end point}

There was a significant reduction from baseline in sUA at 4 weeks in each lesinurad group compared with placebo $(\mathrm{p}<0.0001$, each comparison; figure 2). In the lesinurad 200, 400 and $600 \mathrm{mg}$ groups, the arithmetic mean percent reduction in sUA was $16 \%, 22 \%$ and $30 \%$, respectively, versus a $3 \%$ increase with placebo.

\section{Secondary efficacy end points}

The proportion of patients achieving sUA $<6 \mathrm{mg} / \mathrm{dL}$ at 4 weeks was significantly greater in each lesinurad group versus placebo $(p<0.0001$, each comparison; figure 3$)$. In the non-responder imputation analysis (ITT population), 63\%, 73.8\% and $79.2 \%$ of patients receiving lesinurad 200, 400 and $600 \mathrm{mg}$, respectively, achieved sUA $<6 \mathrm{mg} / \mathrm{dL}$ versus $25 \%$ receiving placebo $(p<0.0001$, each comparison vs placebo). Results were similar using the LOCF analysis (data not shown). The proportions of patients with sUA $<5$ and $<4 \mathrm{mg} / \mathrm{dL}$ were also significantly higher with lesinurad versus placebo (ITT, non-responder imputation analysis and LOCF analysis; data not shown).

In the ITT population, mean percent change from baseline in renal UA clearance at 4 weeks was significantly higher with lesinurad $(200 \mathrm{mg}, 43.7 \%$; $400 \mathrm{mg}, 84.9 \%$; $600 \mathrm{mg}, 118.8 \%)$ versus placebo $(8 \% ; \mathrm{p}<0.05$, each comparison), as was mean percent change from baseline in FE of urine UA at 4 weeks (200 mg, 50.7\%; $400 \mathrm{mg}, 110.8 \%$; $600 \mathrm{mg}, 129 \%$ ) versus placebo (5.3\%; $\mathrm{p}<0.05$, each comparison).

\section{Subgroup analyses}

Mean percent reduction from baseline sUA was significantly greater with each lesinurad dose compared with placebo in all subgroups, except for baseline sUA $\geq 10 \mathrm{mg} / \mathrm{dL}$ (table 2). This subgroup generally included the lowest number of patients $(n=1$ or $n=2)$ for each dose.

\section{Pharmacokinetics}

Trough plasma concentrations tended to increase with dose in patients with $\mathrm{CrCl} \geq 90 \mathrm{~mL} / \mathrm{min}$ (normal renal function) and showed no consistent differences versus patients with $\mathrm{CrCl} 60$ $89 \mathrm{~mL} / \mathrm{min}$ (mild renal insufficiency; see online supplementary table S1). There was an apparent increase in trough plasma concentrations in patients with $\mathrm{CrCl} 30-59 \mathrm{~mL} / \mathrm{min}$ (chronic kidney

\begin{tabular}{|c|c|c|c|c|}
\hline Characteristic* & $\begin{array}{l}\text { Lesinurad } 200 \mathrm{mg}+\text { allopurinol } \\
(\mathrm{n}=46)\end{array}$ & $\begin{array}{l}\text { Lesinurad } 400 \mathrm{mg}+\text { allopurinol } \\
(\mathrm{n}=42)\end{array}$ & $\begin{array}{l}\text { Lesinurad } 600 \mathrm{mg}+\text { allopurinol } \\
(\mathrm{n}=48)\end{array}$ & $\begin{array}{l}\text { Placebo+allopurinol } \\
(\mathrm{n}=72)\end{array}$ \\
\hline Men, n (\%) & $44(95.7)$ & 41 (97.6) & $48(100)$ & 71 (98.6) \\
\hline \multicolumn{5}{|l|}{ Race, n (\%) } \\
\hline White & $42(91.3)$ & $39(92.9)$ & $40(83.3)$ & $67(93.1)$ \\
\hline Asian & $2(4.3)$ & 0 & $3(6.3)$ & $1(1.4)$ \\
\hline Native Hawaiian or Pacific Islander & 0 & $1(2.4)$ & $1(2.1)$ & $1(1.4)$ \\
\hline $\mathrm{BMI}, \mathrm{kg} / \mathrm{m}^{2}$ & $32.7(5.5)$ & $32.3(5.1)$ & $33.1(4.7)$ & $31.9(4.8)$ \\
\hline Years since gout diagnosis & $10.2(9.3)$ & $6.7(6.1)$ & $8(6.2)$ & $7.3(6.3)$ \\
\hline Flares in last 12 months & $4.1(3.8)$ & $3.5(2.6)$ & $4.2(3.9)$ & $4.2(3.7)$ \\
\hline $30-59 \mathrm{~mL} / \mathrm{min}$ & $3(6.5)$ & $2(4.8)$ & $2(4.2)$ & $2(2.8)$ \\
\hline$<30 \mathrm{~mL} / \mathrm{min}$ & 0 & 0 & 0 & 0 \\
\hline
\end{tabular}

*Values are mean (SD) unless otherwise specified.

tLast SUA value recorded before the first dose of blinded lesinurad or placebo.

¥Based on Cockcroft-Gault formula using ideal body weight; using actual weight, 79\%-95\% of patients (across groups) had normal renal function.

$\mathrm{BMI}$, body mass index; $\mathrm{CrCl}$, creatinine clearance; ITT, intention-to-treat; sUA, serum urate. 


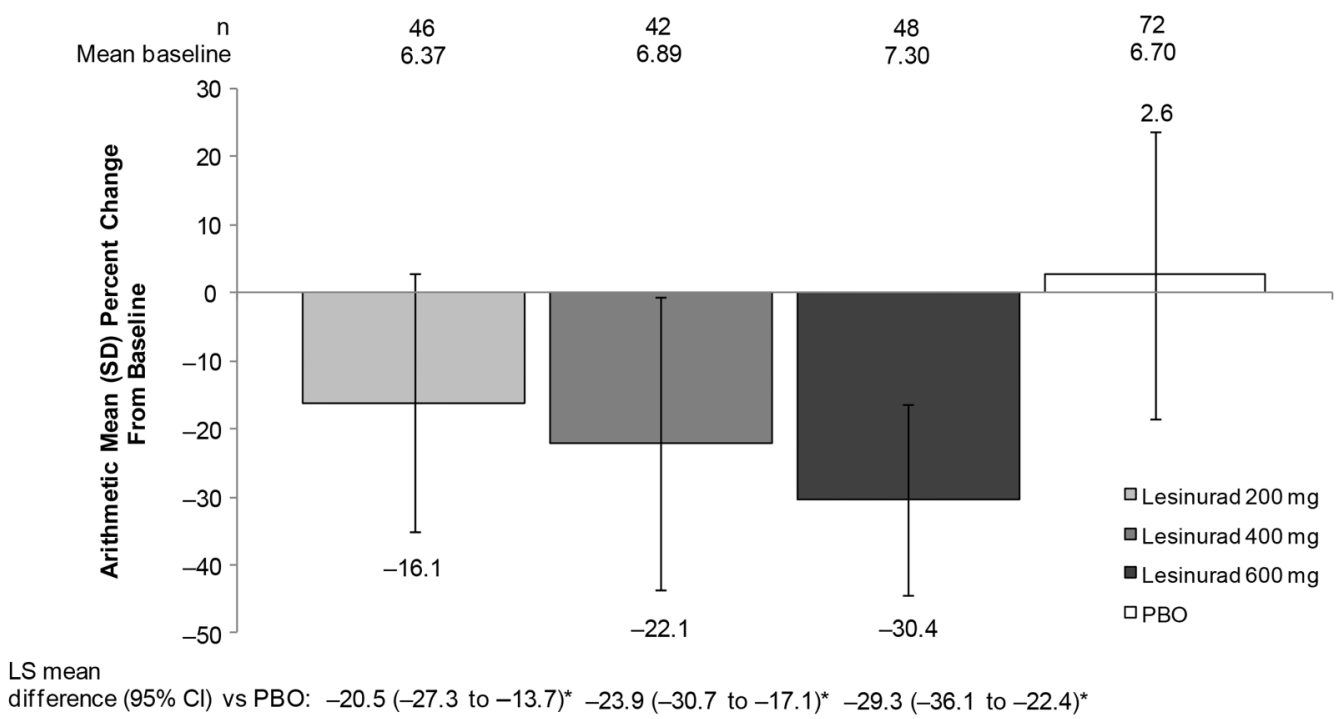

Figure 2 Arithmetic mean (SD) percent change from baseline in sUA concentration at 4 weeks (ITT population). LS mean differences compare each lesinurad treatment group with the pooled placebo group with an analysis of covariance model with effects for treatment group and baseline sUA. ${ }^{*} p<0.0001$ versus PBO. ITT, intention-to-treat; LS, least squares; PBO, placebo; sUA, serum urate.

disease stage 3), but group sizes were small (see online supplementary table S1). The PK substudy included 33 lesinurad patients (200 mg, $\mathrm{n}=10 ; 400 \mathrm{mg}, \mathrm{n}=9 ; 600 \mathrm{mg}, \mathrm{n}=14$ ) and 21 placebo patients. Serial PK analyses are shown in online supplementary table S2. Median plasma lesinurad exposure (area under the plasma concentration-time curve from time zero up to the dosing interval tau of $24 \mathrm{~h}\left(\mathrm{AUC}_{0-24}\right)$ ) was
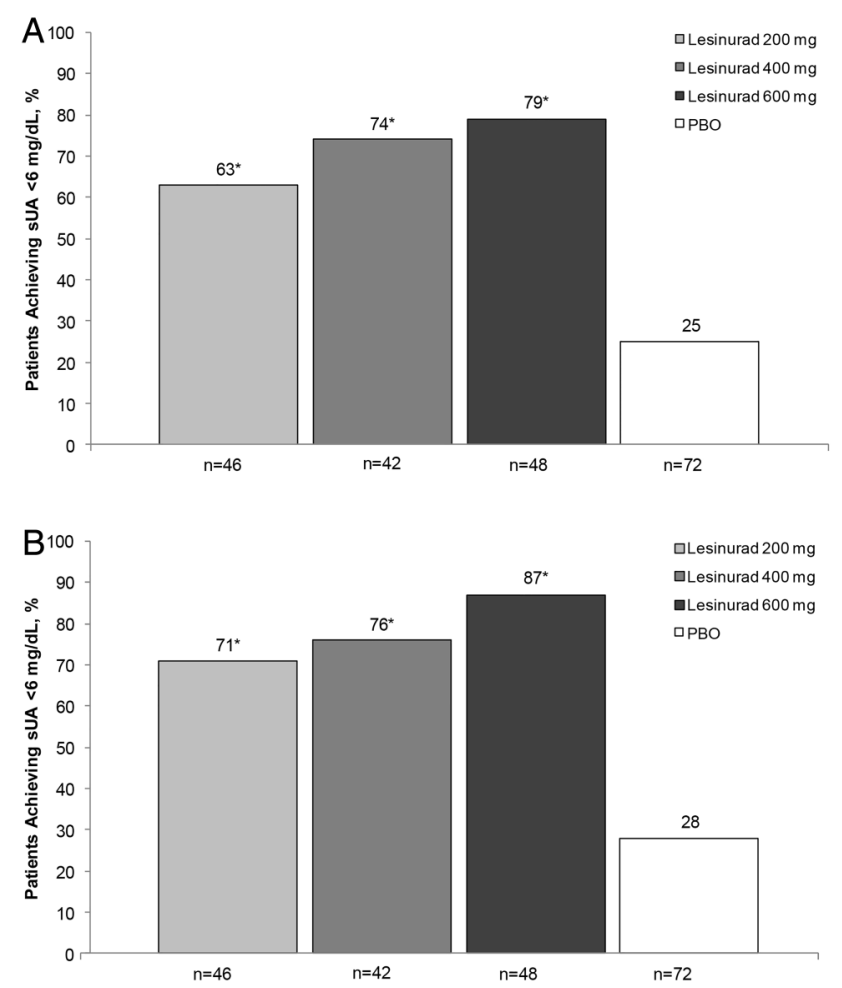

Figure 3 Proportion of patients achieving sUA $<6 \mathrm{mg} / \mathrm{dL}$ at 4 weeks in the (A) ITT population with non-responder imputation analysis and (B) ITT population (LOCF analysis). ITT, intention-to-treat; LOCF, last observation carried forward; PBO, placebo; sUA, serum urate. ${ }^{*} p<0.0001$ versus PBO. approximately proportional to dose in patients with $\mathrm{CrCl} \geq 90$ or $60-89 \mathrm{~mL} / \mathrm{min}$.

Lesinurad treatment did not affect plasma PK or urinary excretion of allopurinol (data not shown). Plasma exposure to oxypurinol decreased by approximately one-third with lesinurad. After administration of $300 \mathrm{mg}$ allopurinol, geometric mean ratios (90\% CI) for oxypurinol maximum observed plasma concentration (day 13/day -1 (baseline)) were 118\% (90\%-156\%) for placebo, 76\% (56\%-104\%) for lesinurad $200 \mathrm{mg}$ and 64\% (47\%-87\%) for lesinurad $400 \mathrm{mg}$; those for oxypurinol $\mathrm{AUC}_{0-24}$ (day 13/day -1 (baseline)) were 116\% (91\%-148\%) for placebo, 68\% (47\%-98\%) for lesinurad $200 \mathrm{mg}$ and $68 \%$ (58\%-78\%) for lesinurad $400 \mathrm{mg}$. Lesinurad had no effect on plasma colchicine exposure.

\section{Safety and tolerability}

The proportions of patients with $\geq 1$ TEAE were similar across groups (table 3 ). The most common TEAEs were gout flare, arthralgia, headache and nasopharyngitis. Gout flare was the most common TEAE considered possibly related to study treatment; increased lipase was the only other TEAE considered possibly related to treatment and occurring in $>1$ patient on lesinurad (400 mg, $\mathrm{n}=2$ ).

Most TEAEs were mild or moderate in severity; there were no serious AEs or deaths. There was one TEAE-related discontinuation with lesinurad $400 \mathrm{mg}$ (elevated lipase) and two with lesinurad $600 \mathrm{mg}$ (abnormal increased creatine kinase, onset before lesinurad and urticaria after first $200 \mathrm{mg}$ dose).

During the double-blind treatment period, treatmentemergent gout flares requiring treatment were reported in $10.9 \%, 23.8 \%$ and $18.8 \%$ of patients receiving lesinurad 200 , 400 and $600 \mathrm{mg}$, respectively, compared with $6.9 \%$ of patients receiving placebo.

The mean change from baseline at 4 weeks for Cr was 0.031, $0.101,0.072$ and $-0.003 \mathrm{mg} / \mathrm{dL}$ for lesinurad 200,400 , $600 \mathrm{mg}$ and placebo, respectively, and for estimated $\mathrm{CrCl}$ was $-5,-0.7,-2.6$ and $0.7 \mathrm{~mL} / \mathrm{min}$, respectively (see online supplementary table S3). With regard to laboratory abnormalities (not considered TEAEs), clinically relevant differences were seen between groups in the proportion of patients with a shift 


\begin{tabular}{|c|c|c|c|c|}
\hline Subgroup & Lesinurad 200 mg+allopurinol & Lesinurad 400 mg+allopurinol & Lesinurad $600 \mathrm{mg}+$ allopurinol & Placebo+allopurinol \\
\hline \multicolumn{5}{|c|}{ Baseline estimated $\mathrm{CrCl}^{*}$} \\
\hline \multirow[t]{2}{*}{$\geq 90 \mathrm{~mL} / \mathrm{min}$} & $n=22$ & $n=19$ & $n=24$ & $\mathrm{n}=28$ \\
\hline & $-15.35(18.96) \dagger$ & $-19.27(24.86) \dagger$ & $-28(14.59) \dagger$ & $2.7(16.33)$ \\
\hline \multirow[t]{2}{*}{$<90 \mathrm{~mL} / \mathrm{min}$} & $n=20$ & $\mathrm{n}=21$ & $n=18$ & $n=38$ \\
\hline & $-15.03(20.19) \dagger$ & $-24.6(18.4) \dagger$ & $-32.32(13.76) \dagger$ & $2.57(24.27)$ \\
\hline \multicolumn{5}{|l|}{ Baseline sUA } \\
\hline \multirow[t]{2}{*}{$<8 \mathrm{mg} / \mathrm{dL}$} & $n=37$ & $n=34$ & $n=32$ & $n=57$ \\
\hline & $-14.4(18.1) \dagger$ & $-21.8(23) \dagger$ & $-27.6(12.8) \dagger$ & $4.6(21.9)$ \\
\hline \multirow[t]{2}{*}{$8-<10 \mathrm{mg} / \mathrm{dL}$} & $n=2$ & $\mathrm{n}=4$ & $\mathrm{n}=7$ & $\mathrm{n}=8$ \\
\hline & $-34.8(24.78) \dagger$ & $-25.3(8.8) \dagger$ & $-44.9(11.59) \dagger$ & $-9.59(8.1)$ \\
\hline \multirow[t]{2}{*}{$\geq 10 \mathrm{mg} / \mathrm{dL}$} & $n=1$ & $\mathrm{n}=2$ & $\mathrm{n}=2$ & $\mathrm{n}=1$ \\
\hline & -43.5 (NA) & $-19.9(20.3)$ & $-23(14)$ & -12.9 (NA) \\
\hline \multirow[t]{2}{*}{$\geq 8 \mathrm{mg} / \mathrm{dL}$} & $\mathrm{n}=3$ & $n=6$ & $n=9$ & $n=9$ \\
\hline & $-37.67(18.19) \dagger$ & $-23.5(11.67) \dagger$ & $-40(14.73) \dagger$ & $-9.91(7.67)$ \\
\hline \multicolumn{5}{|c|}{ Initial allopurinol dose } \\
\hline \multirow[t]{2}{*}{$\geq 300 \mathrm{mg}$} & $\mathrm{n}=36$ & $\mathrm{n}=36$ & $\mathrm{n}=31$ & $n=54$ \\
\hline & $-13.62(18.77) \dagger$ & $-21.03(21.83) \dagger$ & $-29.63(15.06) \dagger$ & $3.43(22.84)$ \\
\hline \multirow[t]{2}{*}{$<300 \mathrm{mg}$} & $\mathrm{n}=6$ & $\mathrm{n}=4$ & $\mathrm{n}=11$ & $\mathrm{n}=12$ \\
\hline & $-24.68(21.63) \dagger$ & $-31.44(19.2) \dagger$ & $-30.48(12.24) \dagger$ & $-1.01(10.21)$ \\
\hline
\end{tabular}

*Based on Cockcroft-Gault formula using ideal body weight.

$\mathrm{tp}<0.05$ versus placebo.

$\mathrm{CrCl}$, creatinine clearance; ITT, intention-to-treat; NA, not available; sUA, serum urate.

from a normal $\mathrm{Cr}$ value at baseline to a value above the reference range at week 4 (lesinurad $200 \mathrm{mg}, 2.5 \%$; $400 \mathrm{mg}, 10 \%$; $600 \mathrm{mg}, 7.5 \%$; placebo, $1.6 \%$ ) and the proportion of patients with a shift in Cr maximum Common Terminology Criteria for Adverse Events (National Cancer Institute; NCI-CTCAE V.4) grade from Gr0 to Gr1 (lesinurad $200 \mathrm{mg}, 2.2 \%$; $400 \mathrm{mg}$, $16.7 \%$; $600 \mathrm{mg}, 12.8 \%$; placebo, $4.2 \%$ ). In addition, one patient who received lesinurad $400 \mathrm{mg}$ shifted from Gr0 to Gr2. The percentages of patients with $\geq 1.5 \times$ and $\geq 2 \times$ increases from baseline in serum $\mathrm{Cr}$ were $0 \%$ with lesinurad $200 \mathrm{mg} ; 12.8 \%$ and $5.1 \%$, respectively, with lesinurad $400 \mathrm{mg}$; $19.7 \%$ and $9.1 \%$, respectively, with lesinurad $600 \mathrm{mg}$ and $2.8 \%$ and $1.4 \%$, respectively, with placebo. No clinically relevant changes in vital signs or ECG findings suggesting short-term cardiac safety risk were observed.

\section{DISCUSSION}

The algorithm based on the ACR guidelines for escalation of urate-lowering therapy recommends that, in patients not achieving target sUA after upward titration of an initial XO inhibitor, another should be substituted. ${ }^{5} 6$ This may also be combined with a uricuretic agent that provides a therapeutic increase in UA excretion from the kidney. ${ }^{14}$ Further reduction to sUA $<5 \mathrm{mg} / \mathrm{dL}$ is recommended for severe gout, polyarticular gout and tophaceous gout. ${ }^{5} 6$ Patients refractory to or intolerant of such options are candidates for pegloticase. ${ }^{5}$ Pegloticase is associated with infusion-related reactions, including anaphylaxis, and requires pretreatment with antihistamines and corticosteroids. ${ }^{16}$ Probenecid may be ineffective in patients with chronic renal disease, has associated drug-drug interactions, and requires twice-daily or four-times-daily dosing. ${ }^{18}$ Benzbromarone has limited availability worldwide and is associated with safety issues. Thus, a safe, effective, and potent URAT1 inhibitor would address important needs.

Most cases of gout $(>90 \%)$ are due to inefficient renal tubular UA excretion ${ }^{19}$; patients have a urine UA clearance of
$<6 \mathrm{~mL} / \mathrm{min}$ (normal, $6-11 \mathrm{~mL} / \mathrm{min}$ ). ${ }^{19}$ In clinical trials of allopurinol, $\leq 40 \%$ of patients achieved sUA $<6 \mathrm{mg} / \mathrm{dL} .{ }^{11} 12$ Although the ACR recommends uptitration of allopurinol in such patients, ${ }^{5}$ this is not typically done in clinical practice. ${ }^{20}$ The combination of an SURI and an XO inhibitor may provide additional benefits by directly addressing the defect in most patients, increasing renal excretion and decreasing production of uric acid. This approach might also allow the use of lower $\mathrm{XO}$ inhibitor doses, possibly reducing adverse effects compared with either drug used alone.

In this phase $2 b$, placebo-controlled study in patients with gout requiring therapy additional to allopurinol, the addition of lesinurad 200, 400 and $600 \mathrm{mg} /$ day produced significant, dose-related reductions in sUA from baseline after 4 weeks (the primary end point). Likewise, significant dose-related improvements were observed in the proportion of patients achieving sUA $<6 \mathrm{mg} / \mathrm{dL}$, percent change from baseline in UA clearance, and percent change from baseline in FE of UA. The combination was well tolerated, with $\mathrm{AE}$ incidences generally similar to or lower than with placebo and mild elevations in serum Cr. These findings suggest that lesinurad can be combined with allopurinol effectively regardless of allopurinol dose $(<300 \mathrm{mg}$ or $\geq 300 \mathrm{mg})$ and sUA level $(<8 \mathrm{mg} / \mathrm{dL}$, 8 to $<10 \mathrm{mg} / \mathrm{dL}$ and $\geq 8 \mathrm{mg} / \mathrm{dL}$ ). However, results for patients with sUA $\geq 10 \mathrm{mg} / \mathrm{dL}$ were inconclusive because of the small group size.

Renal function impairment is common in patients with gout. ${ }^{21}$ Subgroup analyses in patients with $\mathrm{CrCl} \geq 90 \mathrm{~mL} / \mathrm{min}$ (normal renal function) and $<90 \mathrm{~mL} / \mathrm{min}$ (renal insufficiency) demonstrated consistent dose-related reductions in sUA levels with addition of lesinurad to allopurinol compared with allopurinol alone. Significant improvements were observed with all lesinurad doses compared with placebo. In the PK substudy, $\mathrm{CrCl} 60$ $89 \mathrm{~mL} / \mathrm{min}$ (chronic kidney disease stage 2) had modest to negligible impact on lesinurad PK. In subgroups with $\mathrm{CrCl} \geq 90 \mathrm{~mL} /$ min (normal renal function) and $\mathrm{CrCl} 60-89 \mathrm{~mL} / \mathrm{min}$, trough 


\begin{tabular}{|c|c|c|c|c|c|}
\hline n (\%) & $\begin{array}{l}\text { Lesinurad } \\
200 \mathrm{mg}+\text { allopurinol }(n=46)\end{array}$ & $\begin{array}{l}\text { Lesinurad } \\
400 \mathrm{mg}+\text { allopurinol }(n=42)\end{array}$ & $\begin{array}{l}\text { Lesinurad } \\
600 \mathrm{mg}+\text { allopurinol }(n=48)\end{array}$ & $\begin{array}{l}\text { Pooled lesinurad } \\
(n=136)\end{array}$ & $\begin{array}{l}\text { Placebo+allopurino } \\
(n=72)\end{array}$ \\
\hline$\geq 1 \mathrm{TEAE}$ & $21(45.7)$ & $20(47.6)$ & $26(54.2)$ & $67(49.3)$ & $33(45.8)$ \\
\hline$\geq 1$ treatment-related TEAE & $1(2.2)$ & $5(11.9)$ & $5(10.4)$ & $11(8.1)$ & $9(12.5)$ \\
\hline$\geq 1 \mathrm{SAE}$ & 0 & 0 & 0 & 0 & 0 \\
\hline Death & 0 & 0 & 0 & 0 & 0 \\
\hline Discontinuation due to TEAE & 0 & $1(2.4)$ & $2(4.2)$ & $3(2.2)$ & $1(1.4)$ \\
\hline \multicolumn{6}{|l|}{ Specific TEAEs* } \\
\hline Gout flare & $10(21.7)$ & $13(31)$ & $15(31.3)$ & $38(27.9)$ & $15(20.8)$ \\
\hline Arthralgia & $3(6.5)$ & $1(2.4)$ & $2(4.2)$ & $6(4.4)$ & $4(5.6)$ \\
\hline Headache & $3(6.5)$ & $2(4.8)$ & $1(2.1)$ & $6(4.4)$ & $1(1.4)$ \\
\hline Nasopharyngitis & $4(8.7)$ & 0 & $1(2.1)$ & $5(3.7)$ & $1(1.4)$ \\
\hline Tendonitis & $2(4.3)$ & 0 & $1(2.1)$ & $3(2.2)$ & 0 \\
\hline Lipase increased & 0 & $2(4.8)$ & 0 & $2(1.5)$ & $1(1.4)$ \\
\hline URI & 0 & 0 & $2(4.2)$ & $2(1.5)$ & 0 \\
\hline Urticaria & 0 & 0 & $2(4.2)$ & $2(1.5)$ & 0 \\
\hline Diarrhoea & $1(2.2)$ & $1(2.4)$ & 0 & $2(1.5)$ & $3(4.2)$ \\
\hline Haematuria & 0 & $1(2.4)$ & 0 & $1(0.7)$ & $3(4.2)$ \\
\hline Back pain & 0 & $1(2.4)$ & 0 & $1(0.7)$ & $2(2.8)$ \\
\hline Dizziness & $1(2.2)$ & 0 & 0 & $1(0.7)$ & $2(2.8)$ \\
\hline Fatigue & 0 & $1(2.4)$ & 0 & $1(0.7)$ & $2(2.8)$ \\
\hline Myalgia & 0 & $1(2.4)$ & 0 & $1(0.7)$ & 0 \\
\hline Nausea & 0 & 0 & 0 & 0 & $2(2.8)$ \\
\hline
\end{tabular}

${ }^{*}$ Reported in $\geq 1$ patient in any group.

$\mathrm{SAE}$, serious adverse event; TEAE, treatment-emergent adverse event; URI, upper respiratory tract infection.

plasma concentrations were similar, and plasma lesinurad exposure was approximately proportional to dose. This was not unexpected, because lesinurad is eliminated primarily by metabolism rather than by renal excretion of unchanged lesinurad (data on file). Thus, lesinurad can be used effectively with allopurinol in patients with chronic kidney disease stage 2 . Because few patients had $\mathrm{CrCl}$ chronic kidney disease stage 3, further studies are needed to understand lesinurad PK in this population.

Although lesinurad had no appreciable effect on allopurinol plasma PK or urinary excretion, it increased renal clearance of oxypurinol and reduced oxypurinol plasma exposure by approximately one-third, similar to the interaction reported previously for benzbromarone and oxypurinol, ${ }^{22}$ which reflects that oxypurinol, like urate, is a substrate of URAT1. ${ }^{23}{ }^{24}$ Despite the reduction in oxypurinol plasma exposure, the sUA-lowering effect of lesinurad added to allopurinol remained robust. Lesinurad did not impact colchicine plasma PK, suggesting it is unlikely to cause loss of response to colchicine or to increase colchicine toxicity.

Combination therapy with lesinurad and allopurinol was generally well tolerated. Cr elevations occurred at higher dose levels. TEAEs were infrequent, not dose related, mostly mild to moderate in severity, and similar between groups. Gout flares were the most common TEAE; this was expected, as they are a known sequela of sUA lowering during initial urate-lowering therapy, despite ongoing prophylaxis. ${ }^{25}$

The limitations of this study include its 4-week duration, small overall size, sequential cohort enrolment and small sizes of some subgroups. These subgroup analyses should be considered exploratory. Larger studies are needed to evaluate the long-term efficacy and safety of lesinurad in a wider range of patients and to more fully evaluate efficacy and safety in patients with renal function impairment.
Lesinurad in combination with allopurinol significantly lowered sUA at 4 weeks versus placebo (allopurinol alone) in patients with gout and an inadequate response to allopurinol alone and was generally well tolerated.

Contributors FP-R contributed to the study design, study conduct, data analysis/ interpretation and writing of the manuscript. JSS contributed to study conduct/data collection, data analysis/interpretation and writing of the manuscript. JNM, MC and CS contributed to the data analysis/interpretation and writing of the manuscript. All authors provided approval of the final draft for submission.

Funding Ardea Biosciences funded this study. Medical writing support for the preparation of this manuscript was provided by Stephanie Leinbach, Nicole Strangman and Judy Fallon from Complete Healthcare Communications with funding from AstraZeneca.

Competing interests FP-R is a consultant for AstraZeneca, Menarini, Novartis, Pfizer and SOBI; he is a speaker for AstraZeneca and Menarini; and he has received investigation grants from the Asociación de Reumatólogos del Hospital de Cruces.

JSS received research support from Ardea Biosciences a member of the AstraZeneca Group, GlaxoSmithKline, Novartis and Bristol-Myers Squibb; and he is a consultant for AstraZeneca, Jazz Pharmaceuticals, Merck, Par Pharma and Novartis. JNM and CS are employees of Ardea Biosciences a subsidiary fully owned by AstraZeneca. $\mathrm{MC}$ is a former employee of Ardea Biosciences.

\section{Patient consent Obtained.}

Ethics approval The study protocol was reviewed and approved by the institutional review board/independent ethics committee at each site, and the study was conducted in accordance with the Declaration of Helsinki and the International Conference on Harmonisation/Good Clinical Practice.

Provenance and peer review Not commissioned; externally peer reviewed.

Data sharing statement For any data request please submit a request to https:/l astrazenecagroup-dt.pharmacm.com/DT/Home

Open Access This is an Open Access article distributed in accordance with the Creative Commons Attribution Non Commercial (CC BY-NC 4.0) license, which permits others to distribute, remix, adapt, build upon this work non-commercially, and license their derivative works on different terms, provided the original work is properly cited and the use is non-commercial. See: http://creativecommons.org/ licenses/by-nc/4.0/ 


\section{REFERENCES}

1 Zhu Y, Pandya BJ, Choi HK. Prevalence of gout and hyperuricemia in the US general population: the National Health and Nutrition Examination Survey 2007 2008. Arthritis Rheum 2011;63:3136-41.

2 Zhang W, Doherty M, Pascual E, et al. EULAR evidence based recommendations for gout. Part I: diagnosis. Report of a task force of the Standing Committee for International Clinical Studies Including Therapeutics (ESCISIT). Ann Rheum Dis 2006;65:1301-11.

3 Terkeltaub R. Update on gout: new therapeutic strategies and options. Nat Rev Rheumatol 2010;6:30-8.

4 Doherty M. New insights into the epidemiology of gout. Rheumatology (Oxford) 2009;48(Suppl 2):ii2-8.

5 Khanna D, Fitzgerald JD, Khanna PP, et al. 2012 American College of Rheumatology guidelines for management of gout. Part 1: systematic nonpharmacologic and pharmacologic therapeutic approaches to hyperuricemia. Arthritis Care Res (Hoboken) 2012;64:1431-46.

6 Zhang W, Doherty M, Bardin T, et al. EULAR evidence based recommendations for gout. Part II: management. Report of a task force of the EULAR Standing Committee for International Clinical Studies Including Therapeutics (ESCISIT). Ann Rheum Dis 2006;65:1312-24.

7 Perez-Ruiz F, Calabozo M, Pijoan Jl, et al. Effect of urate-lowering therapy on the velocity of size reduction of tophi in chronic gout. Arthritis Rheum 2002;47:356-60.

8 Perez-Ruiz F, Herrero-Beites AM. Evaluation and treatment of gout as a chronic disease. Adv Ther 2012;29:935-46.

9 Sherman MR, Saifer MG, Perez-Ruiz F. PEG-uricase in the management of treatment-resistant gout and hyperuricemia. Adv Drug Deliv Rev 2008;60:59-68.

10 KRYSTEXXA. Pegloticase. Bridgewater, NJ: Savient Pharmaceuticals, 2012.

11 Becker MA, Schumacher HR Jr, Wortmann RL, et al. Febuxostat compared with allopurinol in patients with hyperuricemia and gout. N Engl J Med 2005;353:2450-61.

12 Becker MA, Schumacher HR, Espinoza LR, et al. The urate-lowering efficacy and safety of febuxostat in the treatment of the hyperuricemia of gout: the CONFIRMS trial. Arthritis Res Ther 2010;12:R63.
13 Annemans L, Spaepen E, Gaskin M, et al. Gout in the UK and Germany: prevalence, comorbidities and management in general practice 2000-2005. Ann Rheum Dis 2008;67:960-6.

14 Girardet JL, Miner JN. Urate crystal deposition disease and gout-new therapies for an old problem. In: Desai MC, ed. Annual reports in medicinal chemistry. Burlington: Elsevier, 2014:151-64.

15 Diaz-Torné C, Perez-Herrero N, Perez-Ruiz F. New medications in development for the treatment of hyperuricemia of gout. Curr Opin Rheumatol 2015;27: $164-9$.

16 Wallace SL, Robinson H, Masi AT, et al. Preliminary criteria for the classification of the acute arthritis of primary gout. Arthritis Rheum 1977;20:895-900.

17 Sundy JS, Baraf HS, Yood RA, et al. Efficacy and tolerability of pegloticase for the treatment of chronic gout in patients refractory to conventional treatment: two randomized controlled trials. JAMA 2011;306:711-20.

18 AFT. Probenecid. Auckland, New Zealand: AFT Pharmaceuticals, 2009.

19 Fam AG. Gout: excess calories, purines, and alcohol intake and beyond. Response to a urate-lowering diet. J Rheumatol 2005;32:773-7.

20 Sarawate CA, Brewer KK, Yang W, et al. Gout medication treatment patterns and adherence to standards of care from a managed care perspective. Mayo Clin Proc 2006;81:925-34

21 Zhu Y, Pandya BJ, Choi HK. Comorbidities of gout and hyperuricemia in the US general population: NHANES 2007-2008. Am J Med 2012;125:679-87. e671.

22 Müller FO, Schall R, Groenewoud G, et al. The effect of benzbromarone on allopurinol/oxypurinol kinetics in patients with gout. Eur J Clin Pharmacol 1993;44:69-72.

23 Iwanaga T, Kobayashi D, Hirayama $\mathrm{M}$, et al. Involvement of uric acid transporter in increased renal clearance of the xanthine oxidase inhibitor oxypurinol induced by a uricosuric agent, benzbromarone. Drug Metab Dispos 2005;33:1791-5.

24 Colin JN, Farinotti R, Fredj G, et al. Kinetics of allopurinol and oxipurinol after chronic oral administration. Interaction with benzbromarone. Eur J Clin Pharmacol 1986;31:53-8.

25 Borstad GC, Bryant LR, Abel MP, et al. Colchicine for prophylaxis of acute flares when initiating allopurinol for chronic gouty arthritis. J Rheumatol 2004;31:2429-32. 